\title{
METODOLOGÍA DE SOCIALIZACIÓN Y APLICACIÓN DEL PROCEDIMIENTO PARA LA GESTIÓN DE TRÁMITES DEL DERECHO AL MONTEPÍO.
}

\author{
METHODOLOGY OF SOCIALIZATION AND \\ APPLICATION OF THE PROCEDURE FOR THE \\ MANAGEMENT OF PROCEDURES OF \\ THE RIGHT TO THE MONTEPÍO.
}

\author{
Jessica Janeth Aragadbay Ola ${ }^{1}$
}

IESS

Verónica Leonor Peñaloza López²

PUCESA, Ecuador

\section{RESUMEN}

El Montepío es la pensión mensual que entrega el Instituto Ecuatoriano de Seguridad Social (IESS) a viudos, huérfanos, hijos incapacitados para el trabajo o padres del fallecido, que generaron el derecho. Por lo tanto, se aborda el procedimiento para acceder a la información, con un enfoque especial hacia la referencia a las falencias en cuanto al acceso a esta prestación 1 ORCID https://orcid.org/0000-0002-4735-2754, Abogada de la RepúblicadelEcuador, jessica.j.aragadbay.o@ pucesa.edu.ec, (593) 988578542, Secretaria - Abogada del Juzgado Coactivas Instituto Ecuatoriano de Seguridad Social Chimborazo (IESS), Riobamba, Ecuador.

2 ORCID https://orcid.org/0000-0001-8172-5924, Doctora en Contabilidad por la Universidad Nacional de Rosario, vpenaloza@pucesa.edu.ec, (593) 939424584 Docente Investigadora de la Pontificia Universidad Católica del Ecuador (PUCESA), Ambato, Ecuador. y las deficiencias en el servicio al trámite correspondiente, de acuerdo a la opinión de los beneficiarios registrados en el periodo de 2018, hasta abril de 2021 para conocer el número total de beneficiarios para la aplicación de las encuestas correspondientes determinadas a partir de una muestra de población finita.

El objetivo consiste en diseñar una metodología de socialización y aplicación del procedimiento para la gestión de información y trámites del derecho al Montepío en la Unidad Provincial de Pensiones de Chimborazo. Para realizar esta investigación, se emplea un enfoque mixto, con un diseño no experimental, con datos recopilados de fuentes primarias a través de encuestas aplicadas a la muestra de beneficiarios, además 
se aplicó un análisis FODA para la generación de estrategia que permitan consolidar la metodología de socialización, para este caso es pertinente la aplicación de una metodología de planear, hacer, verificar y actuar (PHVA), misma que se efectúa a través de 4 fases a lo largo de un periodo, lo que permite mejorar la gestión de la información y la atención efectiva para quienes requieren adherirse al derecho de Montepío.

\section{PALABRAS CLAVE:}

Derecho al Montepío; procedimiento; beneficiarios; trámite

\section{ABSTRACT}

The Montepío is the monthly pension that the Ecuadorian Social Security Institute (IESS) delivers to widowers, orphans, children disabled for work or parents of the deceased, who generated the right. Therefore, the procedure to access the information is addressed, highlighting the shortcomings in terms of access to this benefit and the deficiencies in the service to the corresponding procedure, according to the opinion of the beneficiaries registered in the period of 2018, until April 2021 to know the total number of beneficiaries for the application of the corresponding surveys determined from a finite population sample.

The objective is to design a methodology for socialization and application of the procedure for the management of information and procedures for the right to Montepío in the Provincial Unit of Pensions of Chimborazo. To carry out this research, a mixed approach is used, with a nonexperimental design, with data collected from primary sources through surveys applied to the sample of beneficiaries, in addition a SWOT analysis was applied to generate a strategy that allows consolidating the methodology of socialization, for this case the application of a
PDCA methodology (plan, do, verify and act) is pertinent, which is carried out through 4 phases over a period, which allows to improve the management of information and effective care for those who need to adhere to Montepío's law.

\section{KEYWORDS:}

Right to Montepío; process; beneficiaries; Procedure

\section{INTRODUCCIÓN}

La prestación de Montepío, como su nombre lo indica alude a las herramientas mediante las que el seguro social dará una respuesta a las víctimas de algún riesgo que se presente de forma imprevista puesto que se apoya en las aportaciones de los afiliados; es un derecho fundamental de todos los afiliados y jubilados de la Seguridad Social General que cumplen con las imposiciones establecidas, convirtiéndose en un medio económico de subsistencia para las familias que han perdido a un familiar en línea directa (García, 2016).

Para Díaz (2017), es de gran interés para el Estado regir los procesos para la realización de los trámites pertinentes en base a lo establecido en la Constitución, Leyes y Reglamentos. Cabe destacar que la Organización Mundial de la Salud (OMS) con el "Piso de Protección Social" se ha encargado de construir un respaldo para que los ciudadanos de los Estados miembros de esta organización, reciban a través de un grupo de políticas, una garantía de acceso a los distintos servicios sociales, según lo que se ha definido nacionalmente, el goce de estos servicios se enfoca en brindar atención inmediata a los grupos vulnerables o prioritarios como lo reconoce la Constitución de la República del Ecuador la misma que protege los derechos fundamentales que le corresponden al ser humano por la sola razón de existir, por lo que se atribuye la responsabilidad al Estado de otorgar el libre acceso a alimentación, educación, salud, 
vivienda, saneamiento, trabajo, seguridad social, asilo y refugio, la protección y asistencia humanitaria.

El Buen Vivir establece que todas aquellas personas las cuales hayan cumplido con el brindar un servicio a una institución deben ser partícipes de todos los beneficios bajo el amparo de lo que establece el Instituto Ecuatoriano de Seguridad Social (IESS).

Cabe destacar que mediante las leyes y reglamentos que rigen el obrar legal del Ecuador se estable al montepío como un claro derecho de todos los afiliados, dentro del Reglamento de Régimen de Transición Seguro Vejez y Muerte se establece en su artículo 16: "Causará derecho a pensión de montepío el jubilado en goce de pensión de invalidez o vejez, o el asegurado activo que al momento de su fallecimiento tuviere acreditadas al menos sesenta (60) imposiciones mensuales o se encontrare en el período de protección del seguro de muerte".

En este sentido, realizar un análisis sobre el manejo de los tramites de montepío y sobretodo en cuanto al manejo de la información y el conocimiento de los usuarios del mismo se vuelve imprescindible, al ser un derecho como ya hemos mencionado previamente que permite que los familiares del fallecido puedan permanecer asegurados económica ante las posteriores consecuencias que representa la muerte de un familiar dentro de hogar, representa claramente el punto de partida para lograr certificar que se puedan cumplir ciertas necesidad fundamentales y así su calidad de vida sea adecuada.

La socialización de información sobre el derecho al Montepío es crucial para garantizar que a nivel social se conozca este derecho que posee el ciudadano afiliado que recibía una pensión por tema de vejez o alguna discapacidad que lo inhabilite, en caso de ser necesario puede exigirlo basándose en la legalidad que lo respalda, a las personas que hayan enviudado les corresponde el sesenta por ciento de la renta y los hijos que existan de la sociedad conyugal recibirán el cuarenta por ciento, esto para garantizar el sustento del grupo familiar en cuanto al tema económico que involucra todos los aspectos del desarrollo vital, como la alimentación, salud, educación, y comunicación.

En base al diagnóstico previo que se describe en la presente investigación, realizada en la provincia de Chimborazo se ha determinado el alto grado de desinformación de los beneficiarios sobre los procedimientos en cuanto a la gestión de información y tramites relacionado para adquirir el derecho al Montepío que otorga el IESS, lo que produce la tardanza en la entrega de esta prestación por parte de la Unidad encargada, por lo que, se produce insatisfacción de los beneficiarios.

\section{METODOLOGÍA}

La presente investigación parte de un enfoque mixto, puesto que emplea datos tanto cuantitativos como cualitativos para lograr desarrollar una metodología de socialización y aplicación del procedimiento para la gestión de información y trámites del derecho al Montepío en la Unidad Provincial de Pensiones de Chimborazo, la cual da respuesta a cómo mejorar el acceso a la información y trámite para acceder al derecho al Montepío en IESSChimborazo. En este sentido, se considera que los datos serán tomados de los registros estadísticos sobre las personas que han ingresado al servicio de montepío durante los años 2018,2019,2020 y abril del 2021.

Se aplica un diseño no experimental ya que no se realizará ningún tipo de intervención sobre los datos, únicamente estos serán analizados para poder adquirir información pertinente para la investigación que se plantea; en base a un proceso de recolección de datos a partir de encuestas, que serán aplicadas a la muestra 
pertinente, de una población finita, se aplica un cuestionario relacionado con las variables de análisis previamente seleccionadas a partir de una línea base de información primaria donde se considera, la calidad de la atención, talento humano, servicio al usuario, y gestión, con las diferentes perspectivas que estas engloban, a partir del empleo de preguntas cerradas, que permiten una respuesta previamente delimitada, que logran facilitar su posterior codificación, se emplean tanto respuesta dicotómicas como de varias opciones dependiente el requerimiento de cada una de las preguntas, acompañadas previamente de preguntas informativas para tener un mayor conocimiento sobre las características de los usuarios a los que atiende el IESS en este servicio (Sampieri, Fernández, \& Baptista, 2010).

La medición de las respuestas obtenidas de las encuestas aplicadas a la muestra seleccionada se da a partir de un escalamiento tipo Likert, el cual permite otorgar un valor de codificación numérica a cada una de las opciones de categoría que se le plantearon al sujeto encuestado, es así que se logra tener una puntuación por participante respecto a cada una de sus respuestas, de acuerdo a la dirección de cada una de las afirmaciones, sea que estas fueran favorables o desfavorables, en el caso de las primeras se les asigna el valor numérico más alto de la escala, y para el caso contrario el valor más bajo según el grado de actitud al que se refiera. Finalmente, con las puntuaciones que se tenga de manera individual, estas se correlacionan con las puntuaciones a escala global en relación a los reactivos planteados, para así formular un instrumento de medición que de paso a un análisis que permita obtener información valida y confiable para el desarrollo de la metodología de socialización y aplicación del procedimiento para la gestión de información y trámites del derecho al Montepío, una vez determinada la perspectiva que tienen los usuarios sobre este servicio. Cabe destacar que para la realización correcta de esta metodología se parte de un análisis de Matriz FODA y SWOT para la posterior aplicación de la metodología PHVA (planear, hacer, verificar y actuar), en cuanto a la normativa ISO establecida para las actividades dentro del sector público.

\section{ESTUDIO DE LA METODOLOGÍA DE SOCIALIZACIÓN Y APLICACIÓN PARA LA GESTIÓN DE TRÁMITES DEL DERECHO AL MONTEPÍO}

Los métodos de información que utilizan las organizaciones modernas para dar a conocer el proceso del trámite para acceder al Montepío, se han transformado en componentes del éxito o fracaso para la toma de decisiones del afiliado respecto al aporte que han venido contribuyendo para su jubilación. Trischler (1998) mencionaba que: el éxito de toda organización depende cada vez más de que sus procesos estén alineados con su estrategia, misión y objetivos.

Es decir que cada proyecto que se ejecute debe poseer las características propias de la institución que la representan dentro de la sociedad y cumplir con la función de encaminar a la institución a cumplir las metas a futuro. Además, los servidores de la institución deben comprender la importancia que tiene su rol dentro del equipo para lograr el alcance de los objetivos institucionales, que forman parte de la construcción de mejorar la calidad del servicio para los afiliados, la atención, la eficacia de los servicios, la rapidez y el nivel de capacitación que posean los profesionales es la imagen de la institución.

Una de las metodologías más adecuadas para establecer una guía en cuanto al manejo del servicio y la información en la que plante el tratadista Nogueira (2002) donde delimita categorías específicas que orientan el desarrollo práctico de una manera veloz, este proceso tiene un aspecto negativo que representa una 
carencia, la no concepción de un momento analítico para la mejora del proceso, en el que se puede valorar el presupuesto de la institución, la imagen social y la forma de organización que tiene el equipo, al hacerlo todo desde un punto de partida inmediato, se originan varios vacíos que son sumamente importantes para que esta pueda seguir un servicio eficiente a los usuarios.

Para realizar de manera correcta una propuesta de mejora para los procesos y las estrategias de desarrollo de proyectos en la institución, se debe tener presente un equipo de trabajo interdisciplinario donde los miembros poseen conocimientos en herramientas de gestión, y así nombrar a un funcionario adecuado como coordinador para la implementación de estos cambios en torno a la atención del usuarios puesto que se debe tener como prioridad el mejoramiento continuo del servicio como tal, pues se debe atender a las necesidades de los afiliados en relación con los aportes que podrán recibir y los costos que puede tener la implementación de estos procesos.

La gestión de procesos involucra la realización de varios períodos para comprobar la calidad de las propuestas planteadas, los cuales dependerán de varios aspectos como: la complejidad del trámite que haya planteado realizar la institución como meta, la experticia de la entidad respecto a esos procesos concretos de mejoramiento, racionalizaciones previas para que se pueda valorar que dicho trámite es viable para que la institución pueda costear con los gastos que correrán al momento de realizar la mejora. Con esta metodología se logra hacer un presupuesto exacto basado en datos reales para que se disminuyan los procesos donde prima la ineficiencia y la institución pueda permitirse al tener como principal objetivo mejorar el servicio al usar como recurso base la capacitación al personal encargado para que la atención que brinden llegue a satisfacer al usuario.
La metodología de simplificación de trámites propuesto por la FUNDES parte de un "enfoque mixto" que va más allá de reinventar los métodos que se encuentran en análisis. La metodología presenta un enfoque "centrado en el proceso", para animar la complejidad de los tramites esta es la herramienta fundamental para simplificar las prácticas cotidianas en la materia, los trámites para el registro, inscripción y ejecución, el cual se complementa con un enfoque "centrado en el uso" que valora el grado de aceptabilidad que ha conseguido la solución en la socialización con los usuarios.

La metodología elaborada por FUNDES puede llegar a ser replicada en varios países que cuentan con contextos sumamente distintos, tanto a nivel regional como internacional. Se trata de una metodología de aplicación general; que puede adaptarse a servicios distintos, y la facilidad de adaptación de esta metodología nos demuestra su gran valor y vuelve más puesto que permite simplificar lo que resulta tedioso para los usuarios y maximizar la calidad y la grata experiencia que se recibirá del servicio (FUNDES \& CEPAL,2007)

La Metodología de Mejoramiento de Procesos (MMP-GMQFD) aplicado abarca aspectos teóricos de los Modelos de Excelencia, el Enfoque de Gestión por Procesos y el Despliegue de la Función de Calidad, ha sido diseñado precisamente con el fin ofrecer una metodología clara respecto a cómo realizar mejoras continuas que permitan a la institución alcanzar niveles de excelencia. La mejora en la gestión de procesos se desarrolló a base de emplear cuatro etapas: La primera se desarrolla al realizar un extenso estudio global; segunda etapa, en la que se realiza el análisis sistemático; tercera, se regulan de todas las actividades; cuarta, se observan los resultados obtenidos, con el fin de poder seleccionar de la manera correcta el proceso adecuado que se usara para mejorar la calidad de servicio que brinda (BERDUGO, 2003). 
El Modelo EFQM es un tipo de guía para gestionar la calidad de la organización del equipo de trabajo para que la atención que reciba el usuario sea la mejor posible al enfocarse en el tipo de criterio que estos presentan; la sensibilización del equipo directivo y de los funcionarios se enfoca en que se considere a los usuarios como la razón de ser del servicio y esto logre de una manera asertiva dejar una huella en la memoria del usuario, para que se pueda ampliar el conocimiento social que se tiene acerca de la entidad, y de esta forma atraer usuarios, al tener como prioridad ofrecer calidad y una experiencia agradable lo que permita que los trabajadores se sientan conformes con los resultados de su esfuerzo.

Para alcanzar la efectividad en las instituciones en cuanto a la gestión de los procesos, se debe tener presente mejorar el desempeño (eficiencia y eficacia) por parte del equipo y como resultado de su trabajo el servicio y posibilitar de forma continua una mejora que permita mantener el prestigio y la confianza de los usuarios, para crear en ellos un sentimiento de fidelidad hacia la institución que cumpliría con el objetivo de trascender de generación en generación al crear un legado en base a las experiencias que se ha obtenido con el servicio que se ofrece, es decir, que el usuario confié.

\section{DELIMITACIÓN CONCEPTUAL MONTEPÍO (SEGURO DE VIDA).}

Es una renta mensual que otorga el IESS, a los viudos y huérfanos o a padres de los afiliados activos o jubilados fallecidos siempre y cuando generaron el derecho a la prestación de Montepío, de manera que, tenga como mínimo sesenta imposiciones mensuales que equivalen a cinco de aportes y se encuentren dentro del periodo de protección (Muñoz, 2015).

\section{PRESTACIONES.}

Un grupo de beneficios o retribuciones que se encuentran respaldados ante la ley como un derecho legítimo de las personas que rinden un trabajo que requiere honorarios, dicho derecho se otorga a los trabajadores por parte de la institución, es la acción a través de la cual se concede algo a otra persona, basándose en un acuerdo que se tiene para comprometer a las dos partes a realizar sus obligaciones (Benavides, 2008)

Dentro del marco legal se encuentra establecido en el Art. 209 de la Ley de Seguridad Social, menciona que cuando se cumpla con la suma de los aportes que se requieren para al momento de su muerte otorgarles a los tutores del derecho familiares del afiliado una pensión que garantice su supervivencia, en el mismo estilo de vida, sea por viudez, orfandad o a los padres de la persona que ha fallecido y recibía la pensión por vejez o invalidez.

\section{TRÁMITE.}

Conjunto de requisitos o pasos reguladas por la Institución que presta el servicio, dentro de un proceso misional y que deben efectuar los usuarios ante una entidad u organismo de la administración pública o particular que ejerce funciones administrativas para acceder a un derecho, ejercer una actividad o cumplir con una obligación (Bertrand, 2019).

\section{PROCEDIMIENTO ADMINISTRATIVO.}

Conjunto de requisitos, pasos o acciones dentro de un proceso que determina una entidad $u$ organismo de la administración pública que ejerce funciones administrativas para permitir el acceso de los ciudadanos, usuarios o grupos de interés a los beneficios derivados de programas o estrategias cuya creación, adopción e implementación es potestativa de la entidad (Gutiérrez. 2017).

\section{RACIONALIZACIÓN DE TRÁMITES.}

Es la implementación de actividades relacionadas con la simplificación, estandarización, 
eliminación, optimización y automatización de trámites y procedimientos administrativos, que busca disminuir costos, tiempos, requisitos, pasos, procedimientos y procesos, mejorar canales de atención, para facilitar a los ciudadanos el acceso a sus derechos de una manera pronta y eficaz (Amín, 2019).

\section{VENTANILLAS ÚNICAS.}

Son plataformas que permiten que un usuario pueda hacer contacto con la institución para la obtención de un servicio, el acceso a un derecho o el pago de una obligación, a partir de la confluencia de una cadena de trámites en un único punto de acceso de cara al usuario. Este único punto de acceso gestiona, de manera integrada, la realización de trámites que están en cabeza de una o varias entidades (Plaza, 2021).

\section{¿QUIÉN TIENE DERECHO A LA PENSIÓN DE MONTEPIOO?}

a) La cónyuge, el cónyuge o conviviente del afiliado o jubilado fallecido.

b) Hijos del afiliado o jubilado fallecido hasta alcanzar los dieciocho (18) años de edad. También tendrán derecho a pensión de orfandad los hijos de cualquier edad incapacitados para el trabajo, solteros viudos o divorciados que se hayan encontrado bajo la protección del sustento económico del afiliado o jubilado fallecido.

c) En caso de que no se presenten los mencionados al derecho del montepío les corresponde a los padres del jubilado o afiliado fallecido siempre y cuando estos hayan vivido a cargo del causante o hayan sido los tutores de esta persona debido a su necesidad de cuidados por invalidez.

Para darle sustento legal es menester sacar a colación el artículo 198 de la Ley de Seguridad Social, los beneficiarios del subsidio otorgado por el derecho al Montepío serán los derechohabientes del fallecido afiliado al
IESS, ya sea con quien una sociedad conyugal y los hijos, en base a esta conexión se podrá reclamar el derecho al montepío una vez que se demuestre en la Unidad encargada del IESS con la documentación pertinente para el trámite. Como lo enuncia el Art 196, en caso de no encontrarse estas personas que constan en primera línea para acceder al derecho de montepío, pasará el subsidio a los padres que hayan estado al cuidado del afiliado antes de su fallecimiento.

Casos en los que se concede el Montepío.

1.- Al fallecimiento del afiliado activo

2.- Al fallecimiento del jubilado en goce de la pensión

Según el artículo 193 de la Ley de Seguridad Social las personas jubiladas que se encuentren en un periodo en el que reciben beneficios económicos por pensión de vejez o invalidez o la persona afiliada activa con sus aportes que al momento de su defunción tuviera en sumatoria 60 imposiciones mensuales como mínimo, serán los causantes del derecho al montepío.

\section{RESULTADOSDELAINVESTIGACIÓN}

Considera la hipótesis propuesta en este trabajo en base al diseño de una metodología de socialización para la gestión de información y trámite del derecho al Montepío, como medio para mejorar el servicio, y bajo las encuestas realizadas a la muestra calculada a partir de los beneficiarios registrados en el periodo comprendido entre los años 2018 hasta abril de 2021, se tiene que han sido aplicadas 257 encuestas, consideración una población promedio de beneficiarios de 993 , esto a través de la determinación del tamaño de la muestra a partir de la fórmula para una población finita, con un nivel de confianza del $95 \%$ correspondiente a un valor de 1,96 y un error de 0,05.

$$
n=\frac{Z^{2} d^{2} N}{(e)^{2}(N-1)+Z^{2} d^{2}}
$$


Donde:

$\mathrm{N}=$ Tamaño de la población

$\mathrm{n}=$ Tamaño de la muestra

e = Error máximo admisible

$d=$ Varianza 0,25

$Z=$ Nivel de Confianza

Una vez tabulada la información recabada, es $100 \%$ correspondiente a beneficiarios de montepío en la Provincia de Chimborazo, a partir de esto y en correlación a las variables de análisis establecidas previamente se propone un análisis a partir de una matriz FODA (Fortalezas, Oportunidad, Debilidades y Amenazas) en cuanto al servicio, para posteriormente poder convalidar estos hallazgos en una matriz SWOT que permitan determinar estrategias claves con respecto a las características del servicio y así poder estructurar la metodología adecuada.

En este sentido, es importante definir una matriz FODA, la cual alude a un análisis sobre una situación particular, para el caso, el servicio de Montepío, esta herramienta permite realizar un estudio puntual sobre una realidad en un periodo de tiempo determinado con el fin de diagnosticar de manera adecuada las condiciones que permitan a la institución continuar con un positivo desarrollo (Chiavenato, 2017).

La matriz de fortalezas-debilidadesoportunidades-amenazas denominada SWOT, es una metodología propuesta por Fred (2013), que consiste en analizar los cuatro tipos de estrategias: FO (fortalezas-oportunidades), DO (debilidades oportunidades), FA (fortalezasamenazas) y DA (debilidades amenazas). De esta manera, se da una relación de los factores externos e internos claves. Para su realización exige muy buen juicio, puesto que no se establecen series de conciliaciones superiores a las demás.
- Estrategias FO: utilizan las fortalezas internas para aprovechar las oportunidades externas.

- Estrategias DO: tienen como objetivo superar las debilidades internas al aprovechar al máximo las oportunidades externas que se presentan para la institución.

- Estrategias FA: utilizan las fortalezas para evitar o reducir el efecto de las amenazas externas.

- Estrategias DA: son tácticas defensivas dirigidas a la reducción de las debilidades internas y a evitar las amenazas externas.

De acuerdo a la opinión de los beneficiarios sobre la calidad de la atención, en concordancia con el profesionalismos de los funcionarios, seguimiento del trámite y tiempo que este toma; calidad del talento humano en torno a la capacitación y conocimiento de los funcionarios; servicio al usuario, respecto a la información preliminar, procesos y requisitos para realizar el trámite correspondiente; gestión en cuanto a convenios de cooperación de la institución con otras entidades y el manejo de tecnologías fueron planteadas 17 preguntas que han permite recabar la información pertinente para la evaluación de acuerdo a las matrices antes planteadas, en tal virtud a continuación se describen los hallazgos encontrados. 
Tabla 1: Usuarios Activos del Servicio de montepío en Chimborazo por categorías.

\begin{tabular}{lcc}
\hline \multicolumn{1}{c}{ Categoría } & Encuestados & Porcentaje \\
\hline Viudo/ Viuda. & 236 & $84,9 \%$ \\
Hijo/hija del fallecido. & 11 & $11,5 \%$ \\
Padres que se encontraban a cargo del fallecido. & 9 & $3,2 \%$ \\
Hijo/hija beneficiario que presenta algún tipo de discapacidad. & 1 & $0,4 \%$ \\
\hline Total & 257 & $100 \%$ \\
\hline
\end{tabular}

Fuente: Elaboración propia según fuente directa.

En la tabla 1 , el $100 \%$ (257) es de los encuestados son beneficiarios activos de Montepío en la provincia de Chimborazo, de los cuales el 84,9\% (236) corresponde a la categoría de viudo/ viuda, seguido del $11,5 \%$ (11) que son hijo/hija del fallecido, en tercer lugar, padres que se encontraban a cargo del fallecido con un
$3,2 \%$ (9) y finalmente un $0,4 \%$ (1) en relación a hijo/hija beneficiario que presenta algún tipo de discapacidad; así se puede determinar la categoría de cada uno de los beneficiarios donde se destacan los conyugues o convivientes del fallecido en su gran mayoría.

Tabla 2: Calificación de la calidad del servicio.

\begin{tabular}{lcc}
\hline \multicolumn{1}{c}{ Categoría } & Encuestados & Porcentaje \\
\hline Excelente & 7 & $2,5 \%$ \\
Buena & 57 & $20,5 \%$ \\
Regular & 153 & $55 \%$ \\
Mala & 52 & $18,7 \%$ \\
Pésima & 9 & $3,2 \%$ \\
\hline Total & 257 & $100 \%$ \\
\hline
\end{tabular}

Fuente: Elaboración propia según fuente directa.

Según la tabla 2, la calificación de los encuestados con respecto a la calidad del servicio brindada por los empleados del IESS, con un 55\% se establece como regular seguida del $20,5 \%$ que siente que esta es buena, el 18,7\% como mala, el $3,2 \%$ y el $2,5 \%$ correspondiente a la pésima y excelente respectivamente.

Tabla 3: Calificación del conocimiento de los funcionarios.

\begin{tabular}{lcc}
\hline \multicolumn{1}{c}{ Categoría } & Encuestados & Porcentaje \\
\hline Muy bueno & 5 & $1,8 \%$ \\
Bueno & 60 & $21,6 \%$ \\
Medio & 132 & $47,5 \%$ \\
Malo & 75 & $27 \%$ \\
Muy malo & 6 & $2,2 \%$ \\
\hline Total & 257 & $100 \%$ \\
\hline
\end{tabular}

Fuente: Elaboración propia según fuente directa. 
En relación al reactivo anteriormente citado la evaluación del conocimiento de los funcionarios de acuerdo a la ejecución del trámite de Montepío, mismo que ha sido catalogado en la tabla 3 , con un $47,5 \%$ como medio, seguido del $27 \%$ como malo, un $21,6 \%$ para bueno,
$2,2 \%$ muy malo y $1,8 \%$ muy bueno, en este sentido se puede determinar que el servicio en la institución es regular esto debido además de que el conocimiento de los funcionarios es medio o malo según la percepción de los usuarios, sin embargo, este puede ser mejorado a través de las estrategias que se planteen más delante.

Tabla 4: Calificación del tiempo que tardan los trámites.

\begin{tabular}{lcc}
\hline \multicolumn{1}{c}{ Categoría } & Encuestados & Porcentaje \\
\hline Ágiles & 3 & $1,1 \%$ \\
Acertadas & 44 & $15,8 \%$ \\
Tediosas & 171 & $61,2 \%$ \\
Tardías & 61 & $21,9 \%$ \\
\hline Total & 257 & $100 \%$ \\
\hline
\end{tabular}

Fuente: Elaboración propia según fuente directa.

La tabla 4, determina las opiniones en cuanto al tiempo que se demoran las gestiones correspondientes, se encuentran en su mayoría con una tendencia hacían describirlas como tediosas con el $61,2 \%$ de los encuestados, se tiene además que el $21,9 \%$ siente que la gestiones que se deben realizar para acceder al servicio son tardías y el $15,8 \%$ piensa que estas han sido acertadas, solamente el $1,1 \%$ (3) las perciben como ágiles.

Tabla 5 : Inconvenientes con los funcionarios del IESS al realizar el trámite.

\begin{tabular}{lcc}
\hline \multicolumn{1}{c}{ Categoría } & Encuestados & Porcentaje \\
\hline Nunca he tenido inconveniente. & 77 & $27,7 \%$ \\
He tenido un inconveniente. & 171 & $61,5 \%$ \\
Más de dos inconvenientes. & 30 & $10,8 \%$ \\
\hline Total & 257 & $100 \%$ \\
\hline
\end{tabular}

Fuente: Elaboración propia según fuente directa.

De la tabla 5 , se destaca que el $61,5 \%$ de los encuestados que representan a 171 beneficiarios han tenido algún tipo de inconveniente con un funcionario del IESS a cargo de su trámite, lo cual también deja ver una deficiencia que presenta el servicio en cuando a la satisfacción de las necesidades del usuario, el $27,7 \%$ ha tenido varios inconvenientes con el personal a lo largo del desarrollo de su trámite y solamente el $10,8 \%$ no ha presentado ningún inconveniente. A pesar de esto a continuación se presenta la opinión de los encuestados respecto al personal encargado, en torno a si este es pertinente en relación a su puesto de trabajo. 
Tabla 6: Estimación del personal encargado.

\begin{tabular}{lcc}
\hline \multicolumn{1}{c}{ Categoría } & Encuestados & Porcentaje \\
\hline Muy propicio & 10 & $3,6 \%$ \\
Propicio & 168 & $60,4 \%$ \\
Poco propicio & 100 & $36 \%$ \\
\hline Total & 257 & $100 \%$ \\
\hline
\end{tabular}

Fuente: Elaboración propia según fuente directa.

En relación al reactivo planteado sobre, si el personal es propicio, el $60,4 \%$ de los beneficiarios expresó que el personal encargado de montepío en Chimborazo es el propicio para llevar a cabo este procedimiento, sin embargo, el $36 \%$ percibe como poco propicio para la realización de estas actividades al personal que presta sus labores en las instalaciones de la institución pública según detalle de la tabla 6.

Tabla 7: Manejo de los canales de información y gestión de la calidad.

\begin{tabular}{|c|c|c|}
\hline Categoría & Encuestados & Porcentaje \\
\hline \multicolumn{3}{|c|}{ Capacitación } \\
\hline Si & 210 & $75,5 \%$ \\
\hline No & 68 & $24,5 \%$ \\
\hline Total & 257 & $100 \%$ \\
\hline \multicolumn{3}{|c|}{ Gestión Tecnológica } \\
\hline $\mathrm{Si}$ & 203 & $73 \%$ \\
\hline No & 75 & $27 \%$ \\
\hline Total & 257 & $100 \%$ \\
\hline
\end{tabular}

Fuente: Elaboración propia según fuente directa.

Los mencionado previamente se enlaza con el siguiente reactivo en torno a la capacitación de los funcionarios según tabla 7 , donde el 95,3\% de los encuestados respondió que sí es una necesidad para mejorar el servicio que se ofrece que se capacite a los funcionarios, ligado además a que los canales de información por los cuales se accede a los requerimientos para montepío en un $75,5 \%$ no satisfacen las dudas del usuario, cabe considerar que las plataformas tecnológicas se han volcado como una herramienta importante y sencilla para el acceso general de los beneficiarios estas no han sido manejada de manera efectiva puesto que el $73 \%$ percibe que la gestión tecnológica en relación al ingreso de las solicitudes de montepío no es sencilla, esto puede darse a diversos factores entre los que se tienen además la nula capacitación de los usuarios sobre el manejo de la plataforma correspondiente, al dar un punto focal de las debilidades del servicio de montepío en Chimborazo, al abrirse paso lento en un mercado que se vuelca hacia las Tics (Tecnologías de la Información y Comunicación). 
Tabla 8 : Percepción del usuario sobre aspectos importantes del trámite de Montepío.

\begin{tabular}{lcc}
\hline \multicolumn{1}{c}{ Categoría } & Encuestados & Porcentaje \\
\hline Le otorga la seguridad & Seguimiento del trámite \\
No ha obtenido información & 72 & $25,9 \%$ \\
\hline Total & 206 & $74,1 \%$ \\
\hline \multicolumn{3}{c}{ Actitud de los funcionarios } \\
\hline $\mathrm{Si}$ & 237 \\
$\mathrm{No}$ & 41 & $100 \%$ \\
\hline Total & 257 & $85,3 \%$ \\
\hline $\mathrm{Si}$ & Número de funcionarios \\
$\mathrm{No}$ & 30 & $14,7 \%$ \\
\hline Total & 248 & $100 \%$ \\
\hline \multicolumn{3}{c}{257} \\
\hline $\mathrm{Si}$ & $\mathbf{2 5 7}$ & $89,2 \%$ \\
$\mathrm{No}$ & 176 \\
\hline Total & 102 & $100 \%$ \\
\hline
\end{tabular}

Fuente: Elaboración propia según fuente directa.

En cuanto al seguimiento que le dan los funcionarios a cada uno de los trámites los usuarios respondieron según la tabla 8 , en un $74,1 \%$ que no se ha obtenido información acerca del proceso, por lo cual se siente un abandono por parte de la entidad hacia los beneficiarios de este servicio, es importan recalcar que a pesar de esta sensación que se deja en la persona, cuando se les pregunto sobre si la actitud de los funcionarios al momento de receptar de forma presencial la solicitud de montepío es empática, al demostrar el respeto frente al proceso que será realizado, el $85,3 \%$ respondió que si, por lo que se puede aseverar que los inconvenientes surgen en las siguientes etapas del trámite, puesto que el $89,2 \%$ considera que la unidad de servicio no cuenta con el número de funcionarios suficientes para dar una atención pronta a los beneficiarios, por lo cual el tiempo de duración se vuelve más largo, sin embargo, el $63,3 \%$ siente que los requerimiento que han sido planteados por la institución son los adecuados para acceder al montepío. 
Tabla 9: Conocimiento de los usuarios sobre aspectos de la Institución y el Montepío.

\begin{tabular}{|c|c|c|}
\hline Categoría & Encuestados & Porcentaje \\
\hline \multicolumn{3}{|c|}{ Convenios de Cooperación } \\
\hline $\mathrm{Si}$ & 71 & $25,5 \%$ \\
\hline No & 207 & $74,5 \%$ \\
\hline Total & 257 & $100 \%$ \\
\hline \multicolumn{3}{|c|}{ Planificación } \\
\hline $\mathrm{Si}$ & 259 & $93,2 \%$ \\
\hline No & 19 & $6,8 \%$ \\
\hline Total & 257 & $100 \%$ \\
\hline \multicolumn{3}{|c|}{ Medios de Información } \\
\hline Redes Sociales & 12 & $4,3 \%$ \\
\hline Páginas web & 109 & $39,2 \%$ \\
\hline Folletos & 30 & $10,8 \%$ \\
\hline Unidad de servicio (presencial) & 127 & $45,7 \%$ \\
\hline Total & 257 & $100 \%$ \\
\hline
\end{tabular}

Fuente: Elaboración propia según fuente directa.

Finalmente, en cuando a la gestión externa que pueda realizar la institución según detalle de la tabla 9 , los usuarios en un $74,5 \%$ tienen un nulo conocimiento sobre convenios de cooperación que vinculen este servicio con otras instituciones, por lo que la deficiente en cuanto a la información oportuna hacia los usuarios es clara, ya que el $45,7 \%$ accede a está acercándose a las instalaciones de forma presencial, seguido del $39,2 \%$ que lo hace a través de la página web, el $10,8 \%$ a través de folletos que se les entrega de manera similar en la institución, cabe destacar que una fortaleza de este servicio se centra en que el $93,2 \%$ considera que la afiliación al IESS permite planificar a largo plazo en cuando a la seguridad de acceso a un rubro que permita satisfacer necesidades primarias, es así, que al ser una obligación de los empleadores afiliar a los trabajadores, la entidad pública se garantiza la cobertura de la afiliación para cumplir con la demanda de servicios por lo cual los procesos 
Tabla 10: Matriz FODA.

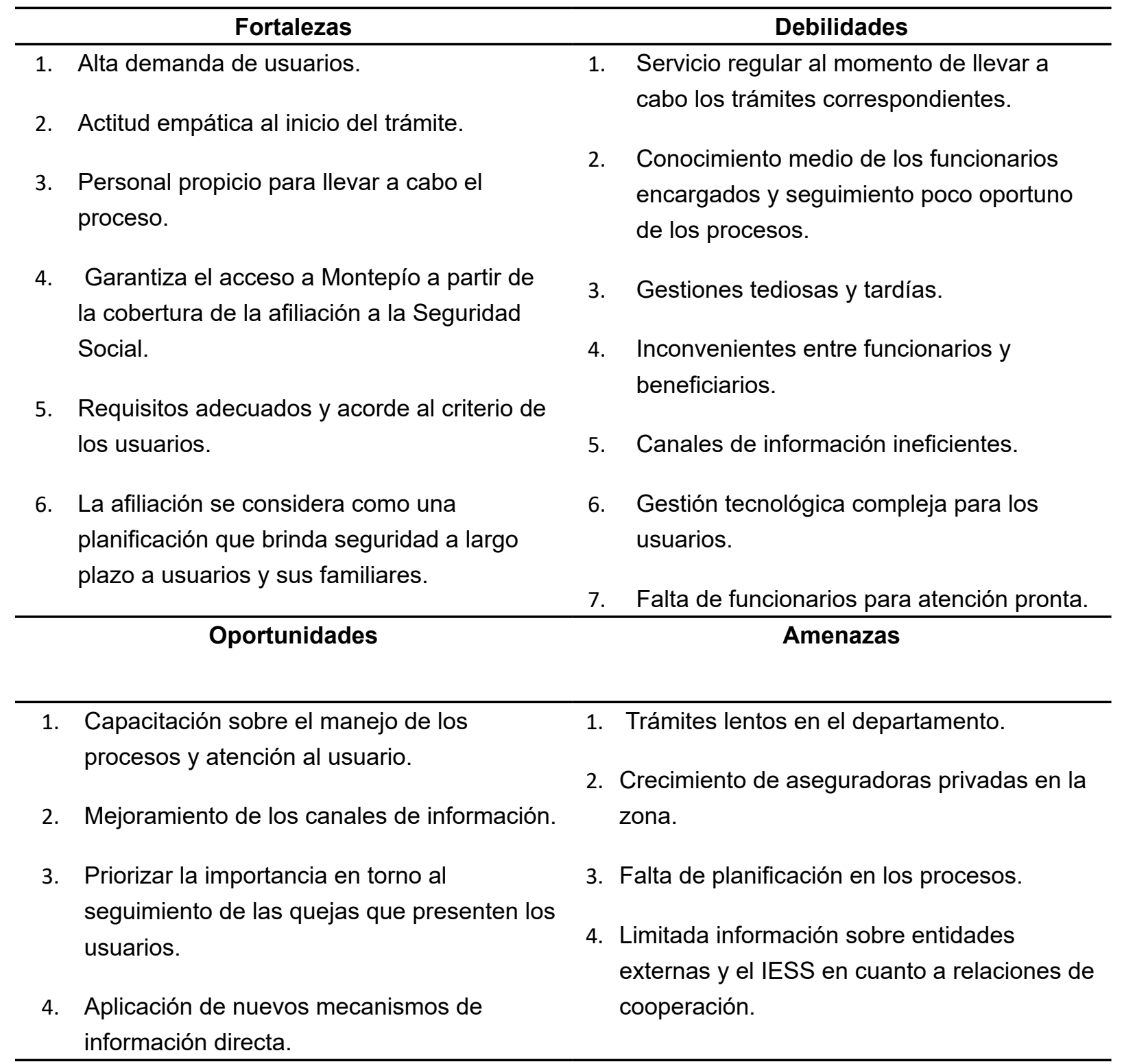

Fuente: Elaboración propia según fuente directa

Se realiza en la tabla 10 , las estrategias en relación a los aspectos que se han podido identificar en la matriz FODA son esenciales para direccionar a la organización hacia una meta u objetivo, además, permiten aprovechar y obtener ventajas ante su entorno; no obstante, se les puede definir como un conjunto de acciones ofensivas y defensivas que tienen la finalidad de establecer características diferenciadoras que ayudan a la sostenibilidad y bienestar de los usuarios (Quero, 2008). 
Tabla 11 : Matriz SWOT.

\section{Estrategias FO}

1. Desarrollar canales de información adecuados para comunicar a la fuerte demanda. (O2-F1)

2. Expandir los mecanismos directos en torno a los requerimientos para acceder al montepío en concordancia a la afiliación. (O4-F4)

3. Capacitación continua del personal en cuanto a atención al cliente y gestión de procesos (O1-F2-F3)

4. Participación activa de los usuarios en relación al planteamiento de mejoras adaptables para su satisfacción. (O3- F5-F6)

\section{Estrategias FA}

1. Fortalecer los canales de acceso a información sobre los beneficios de la afiliación. (F1-A2-A3)

2. Implantar procesos de planificación adecuados en base al cumplimiento de metas y logros. (F5-A3-A1)

3. Promover acuerdos de cooperación entre instituciones ligadas a los procesos que conlleva el montepío. (F6-A4)
Estrategias DO

1. Implementación de planes de evaluación y seguimiento de capacitaciones en relación a la atención al usuario. (O1D2-D4)

2. Creación de fuentes de información acordes para el manejo de los usuarios. (O2-04-D5-D6)

3. Planificación de actividades de acuerdo a cronogramas y horarios previamente establecidos. (O3-D1-D3-D7)

Fuente: Elaboración propia según fuente directa

La matriz SWOT que se detalla previamente, permite establecer lineamientos en torno a la generación de estrategias basadas en las fortalezas, debilidades, amenazas y oportunidades que presenta el IESS específicamente dentro del servicio de Montepío que brinda en la provincia de Chimborazo a los usuarios de lazona, en este sentido las estrategias planteadas se enfocan primordialmente en la mejora de la eficiencia en cuanto a la asignación de tareas específicas, así como implementar análisis de desempeño en base al cumplimiento de objetivos en conjunto con una focalización sobre las necesidades de cada usuario; cabe destacar que la planificación dentro del servicio se vuelve fundamental, tanto de manera interna como externa pues aquí se engloban, el acceso a información, el seguimiento de trámites, y la realización de acuerdos de cooperación con instituciones que puedan fortificar a la institución en esta sección de atención a los usuarios como parte de su derecho por aportar a la seguridad social a través de su trabajo. 
Figura 1: Flujograma de la Metodología.

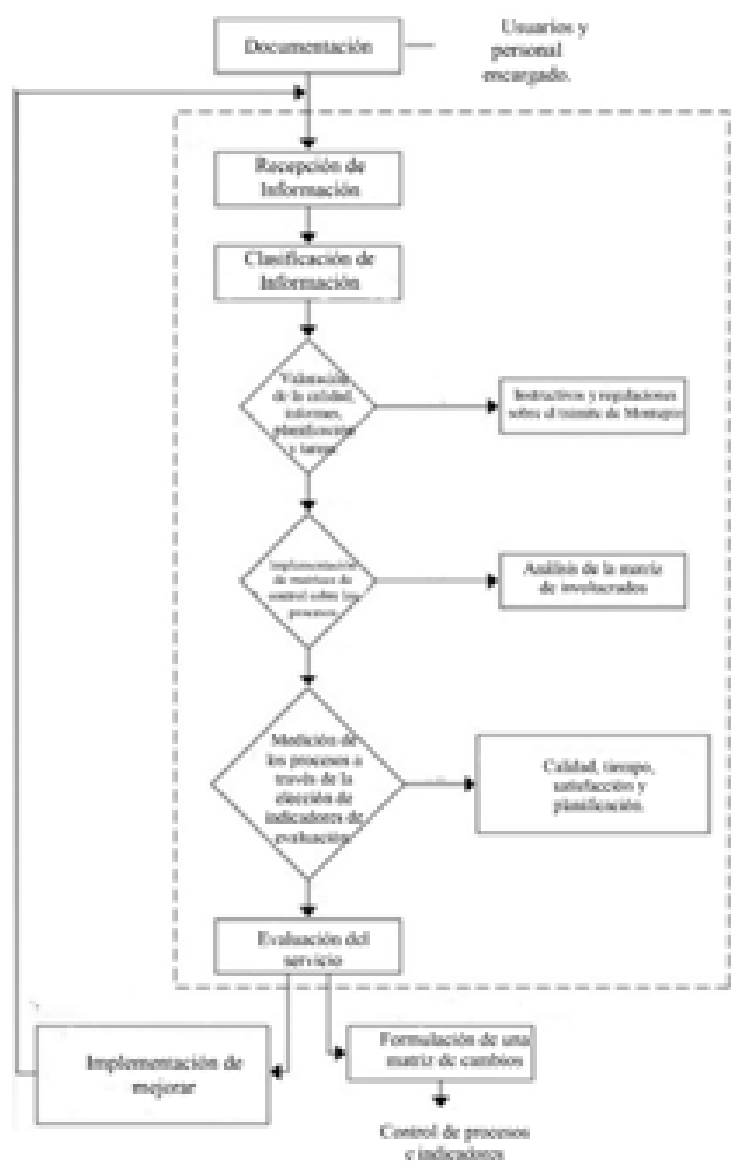

Fuente: Elaboración propia según fuente directa.

La figura 1 muestra el flujograma de pasos a seguir en relación a las estrategias planteadas previamente según la metodología PVHA que se propone, en este sentido se puede mencionar que se debe partir de una valoración de la información que se tiene posterior a la recepción de documentación interna y externa, es decir, de la que se maneja dentro de la institución para el control del servicio y de la que cada uno de los usuarios presenta al momento de acceder a este, para así pasar a un análisis más específico en cada una de las etapas que se deben llevar a cabo, así mismo se entabla la necesidad de una evaluación en base a indicadores claves sobre calidad, tiempo, satisfacción y planificación, variables que fueron analizadas previamente dentro de los reactivos de las encuestas y en donde los usuarios respondieron que existían deficiencias, en este sentido se puede realizar una matriz de cambios para controlar los procesos o en el caso de que los procesos a mejorar sean sencillos se lo haga de forma individual.

En cuanto a la metodología a implementar para la mejora de los procesos con respecto al acceso a información sobre el servicio de Montepío se ha visto pertinente la aplicación de una PHVA, definida como un proceso a través del cual se planea, hace, verifica y actúa, en otras palabras, se debe documentar la información necesaria para los usuarios, para posteriormente implementar las medidas para que estos puedan acceder a la mismas, medir su efecto y el alcance que ha tenido para así determinar 
posibles mejoras a futuro para satisfacer las necesidades de los beneficiarios en cuanto al deseo de adquirir información (Serna, 2000).

Este ciclo también denominado como fases de gestión por procesos continuas, dividas en 4 fases importantes, las cuales se describen a continuación:

Fase 1: Documentar, se realiza un detalle de todos los documentos que permiten organizar la gestión en cuanto al servicio que se pretende prestar, además de la materialización de lineamientos claros que permitan mantener el compromiso en cuanto a la calidad de los procedimientos al tener como base primordial una organización adecuada de cada proceso a llevar a cabo.

Como un apoyo para que la metodología desarrollada tenga una mayor fundamentación en cuanto a las necesidades y requerimiento del usuario, se emplea como guía de su ejecución las normas ISO 9000:2008, mismas que se describen como estándares genéricos que permiten garantizar la calidad del servicio o producto que brinda una organización hacia el público, en base a criterios como los fundamento y vocabulario empleados para dar a conocer información relevante, la calidad de la gestión, los requisitos y las directrices que se hayan desarrollado en torno a las mejoras de desempeño, al tomar en consideración al cliente o usuario, el liderazgo desde los diferentes niveles jerárquicos, el tipo de participación que realice el personal, enfoque tanto del proceso como de la gestión que engloba el servicio, las mejoras duraderas establecidas en relación a la toma de decisiones adecuadas con el fin de fundamentar relaciones beneficiosas entre las partes involucradas (Zaldumbide,2019)

En este sentido se detallan sugerencias como guía para la elaboración de los que se deben incluir en los documentos que describen los lineamientos de acuerdo al compromiso con la calidad de la oferta de este servicio al usuario:

- Manual de valoración de calidad, procesos.

- Especificaciones de cada uno de los documentos para la gestión.

- Informe administrativo en conformidad con las actividades realizadas.

- Planificaciones directivas en base a instructivos y regulaciones sobre todos los aspectos involucrados dentro del trámite de Montepío.

- Delimitación de tareas.

Fase 2: Implementar, pretende la implementación de matrices de control sobre los procesos llevados a cabo para lograr una estandarización de los documentos necesarios para hacer llegar la información al usuario, así también una valoración rápida de los lineamientos planteados previamente en la fase anterior.

- $\quad$ Análisis de una matriz de involucrados que permita conocer las características, funciones y necesidades de cada uno de estos.

- $\quad$ Elaboración de matrices de control de procesos

- Estandarización de documentación permitente en torno al manejo oportuno de la información.

Fase 3: Medir, a través de indicadores para la medición de datos sobre el funcionamiento de cada uno de los procesos que se deben llevar a cabo para la materialización del trámite en cuestión, al partir de la determinación de variables en cuanto al desempeño, el impacto, importancia, funcionalidad y factibilidad de lo que ha sido materializado. 
- Elección de indicadores a valorar de acuerdo a cada uno de los aspectos determinados previamente en las fases 1 y 2 . Esto se puede realizar a través de una matriz de caracterización que permita valorar cada uno de los indicadores elegidos, puesto que permite la gestión y sobretodo el control de los procesos que se han llevado a cabo a lo largo de un periodo de acuerdo a los objetivos que fueron planteados.

- $\quad$ Establecer los procesos que necesitan mejoras en relación al nivel de cumplimiento de metas y objetivos que se logró a lo largo del periodo previamente establecido, a través de técnicas de priorización acordes a los indicadores y las actividades desarrollados en todo el proceso.

Fase 4: Mejora, esta es la fase que cierra el ciclo continúo con el proceso de la determinación de mejoras, en base a los cambios que se han establecido como necesarios en torno a las falencias de cada una de las fases anteriores, a través de la cual se pueden realizar cambios paulatinos y mínimos que generen mejoras y lo que se pretende aplicar en esta investigación una metodología que para desarrollar una nueva versión de los procesos. Esta fase puede realizarse a través de una matriz de cambios donde se evalué lo detallado en las fases 2 y 3 , o en el caso de encontrar varias áreas donde se debe mejorar la gestión del servicio, realizar un proyecto de mejora que dé lugar a un cambio sistematizado.

\section{CONCLUSIONES}

- $\quad$ El servicio al Montepío que brinda el Instituto Ecuatoriano de Seguridad Social, es un derecho vigente en las leyes y fundamentos que rigen el comportamiento de las instituciones públicas como una obligación del Estado en cuanto a salvaguardar el Buen Vivir y bienestar de los afiliados a este sistema, por lo que el uso activo de los canales de información a través de una red que permita el libre acceso y manejo de cada uno de los usuarios es clave para mejorar la gestión.

- La falta de capacitación constante de los funcionarios ha generado que los beneficiarios sientan que el servicio que reciben es regular o malo por lo que, mejorar la gestión del personal en cuanto a atención al cliente, seguimiento del trámite, manejo de información, cumplimiento de metas y determinación de tareas es vital para poder agilizar los trámites, puesto que además así la falta de personal determina por los usuarios como un factor principal de la demora en este servicio no repercutirá en la distribución del presupuesto asignado para personal en la institución.

- Las estrategia que han sido detalladas en los resultados permiten resaltar las fortalezas $\mathrm{u}$ oportunidades que tiene la institución y sobretodo la Unidad Provincial de Pensiones de Chimborazo, para brindar el servicio de Montepío, al poner en consideración las debilidades y amenazas detalladas por los usuarios a través de la encuesta aplicada a estos, para así determinar la aplicación de una metodología de tipo PHVA, a través de la cual se planea, hace, verifica y actúa para poder realizar un proceso concreto de fortalecimiento y mejora para eliminar deficiencias de manera permanente y perfeccionar el servicio de forma constante.

\section{REFERENCIAS BIBLIOGRÁFICAS}

Amín, F. (2019, septiembre). SANTIAGO VALENCIA GONZÁLEZ. https://www. eluniversal.com.co/binrepository/ reglamentar-la-racionalizacion-detramites_2190170_20190924190128. pdf

Benavides, A. B. V. (2008). Análisis práctico de las prestaciones de la Seguridad Social (2a. ed, Vol. 1). Valladolid : Lex Nova. 
Bertrand, A., \& Corona, C. (2019). Ley del Seguro Social comentada, correlacionada $y$ tematizada con sus reglamentos y otros ordenamientos (1a ed.). Capture. https:// www.dijuris.com/libro/ley-del-segurosocial-comentada-correlacionada-ytematizada-con-sus-reglamentos-yotros-ordenamientos_37275

Chiavenato, I. (2017). Planeación estratégica: Fundamentos y aplicaciones. Río de Janeiro: McGraw Hill.

Díaz, N. (2017, 1 febrero). INSTITUCIONALIDAD DEL IESS INSTITUTO ECUATORIANO DE SEGURIDAD SOCIAL. Revista Multidisciplinaria de Investigación, 1(1). http://revistaespirales.com/index.php/ es/article/view/8

Fred, D. (2013). Conceptos de administración estratégica. México: Pearson Educación.

FUNDES \& CEPAL. (2007). Simplificación de trámites para la creación de institucións: la experiencia de Fundes. Naciones Unidas. https://core.ac.uk/download/ pdf/38671875.pdf

Galvis Muñoz, G. M., \& Paula Andrea, P. A. (2015). Sistema de pensión público, Colombia y Ecuador. RIDUM.

http://ridum.umanizales.edu.co/xmlui/ handle/20.500.12746/2656

García, J. (2016, 25 junio). Sistema de pensión público, Colombia y Ecuador. RIDUM. https://ridum.umanizales.edu.co/xmlui/ handle/20.500.12746/2656

González Ortega, S. et al. (2015). La Articulación de un Sistema de Seguridad Social. Ministerio Coordinador de Desarrollo Social. Quito, Ecuador. Primera Edición: 23 de diciembre de 2015, pp 154-164. ISBN: 978-9942-22-035-6
Gutiérrez. Revista Derecho del Estado, 38. https://papers.ssrn.com/sol3/ papers.cfm?abstract_id $=3004825$

Hernández Nariño, Arialys; Nogueira Rivera, Dianelys; Medina León, Alberto; Marqués León, Maylin. Inserción de la gestión por procesos en instituciones hospitalarias. Concepción metodológica y práctica. Revista de Administração - RAUSP, vol. 48, núm. 4, octubre-diciembre, 2013, pp. 739-756. Universidade de São Paulo. São Paulo, Brasil

Nogueira, Y. (2002). Fundamentos teóricoconceptuales de la auditoría de procesos. Retos de la Dirección, 14(1), 1-19.

Palma, H. H., Sierra, D. M., \& Arbelaez, D. C. (2016). Enfoque basado en procesos como estrategia de dirección para las institucións de transformación. Saber, ciencia y libertad, 11(1), 141-150.

Quero, L. (2008). Estrategias competitivas: factor clave de desarrollo. Negotium, 4(10), 36- 49. Obtenido de https://www. redalyc.org/pdf/782/78241004.pdf

Reglamento de Régimen de Transición Seguro Vejez y Muerte. Resolución del IESS 100 de 2021. 09 de marzo de 2006 (Ecuador).

Sampieri, R., Fernández, C., \& Baptista, M. (2010). Metodología de la Investigación (Quinta Edición). Mc Graw Hill.

Serna, H. (2000). Planeación y Gestión Estratégica. Bogotá: Ram Editores.

Trischler, W. E. (1998). Mejora del valor añadido en los procesos: ahorrando tiempo y dinero elimi xnando despilfarro (No. 658.01/T83uE). Gestión 2000. 
Zaldumbide, O. (2019, 20 febrero). Metodología para la gestión por procesos, un enfoque para la implementación. Revista de Ciencias de Seguridad y Defensa, 4(7). http://geo1.espe.edu.ec/wp-content/ uploads//2019/03/7art3.pdf 